\title{
Numerical Simulation Research on Flow and Heat Transfer Process of Fuel Nozzle
}

\author{
Yi Zhang ${ }^{\star}$ and Yubin Men \\ AECC Shenyang Engine Research Institute, Shenyang, China \\ ${ }^{*}$ Corresponding author
}

\begin{abstract}
Keywords: Thermal protection, Coke deposition, Fluid-thermal-solid interaction, Numerical
\end{abstract} simulation.

Abstract. This article presents an investigation on the flow and heat transfer process of a gas turbine fuel nozzle with double channel. The fuel which is influenced by low flow velocity and high-temperature air around nozzle has high probability of coke deposition. The coke deposition problem is related with fuel temperature which is determined by flow and heat transfer process. A three-dimensional fluid-thermal-solid interaction numerical simulation solution is applied to acquire the temperature distribution characteristic in fuel nozzle. The numerical simulation result shows that the flow parameters of both air and flue have influence on the temperature distribution characteristic in fuel nozzle.

\section{Introduction}

Fuel nozzle which is able to atomize the fuel by pneumatic action or pressure effect is widely used in gas turbine. In order to improve atomizing effect, fuel nozzles usually have a complex internal fuel passage which is high-occurrence position of coking. In practical working satiation, the fuel is applied to cool lubrication oil and gas turbine accessories as refrigerant and the fuel is heated by the hot air in combustion chamber before atomizing. On account of the factor mentioned above, the analysis on temperature distribution and the coking possibility when designing a nozzle is necessary.

This article presents a fluid-thermal-solid interaction numerical simulation method to evaluate the performance of fuel nozzle. The performance of a simplified fuel nozzle with double channel which has the structure as what is shown in Figure 1 is investigated based on the numerical simulation result.

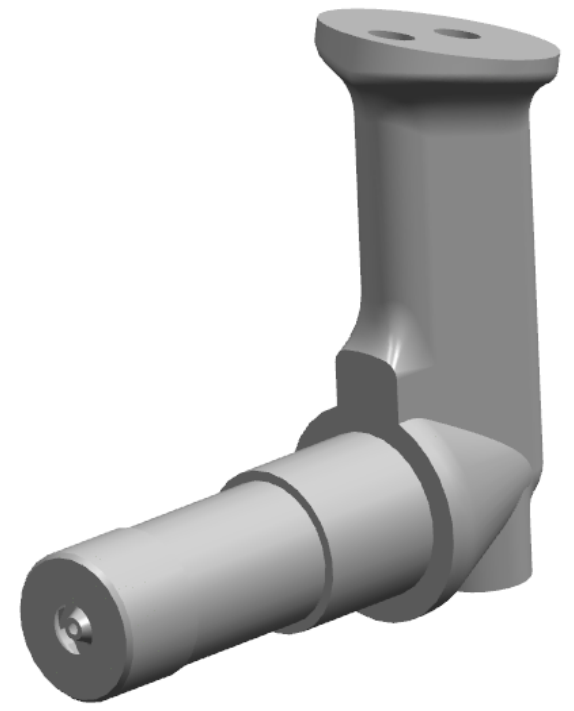

Figure 1. Schematic Diagram of the Simplified Fuel Nozzle 


\section{Calculation Domain and Mesh Generation}

The simplified fuel nozzle is disposed in the passageway of tester in practice. The windward area of simplified fuel nozzle is much less than the flow passage area of tester passageway. In numerical simulation, the numerical simulation process can be simplified to be the problem that is far field flow. The calculation domain is accordingly simplified to be the structure of fuel nozzle in a cubic fluid domain. The surface of cubic field is set as boundary which has steady flow velocity parameter and pressure parameter. The contact surface between simplified fuel nozzle structure and fluid is set as wall boundary which is coupled.

The unstructured gird is applied to divide calculation domain. Approximately 4000000 unstructured girds are used to express the structure of calculation domain. The girds around the structure of fuel nozzle is refined particularly to describe the subtle structure. According to local gird refinement, the unstructured girds which are applied to divide calculation domain are high-quality. The global gird and local refined gird is showed in figure 2 .
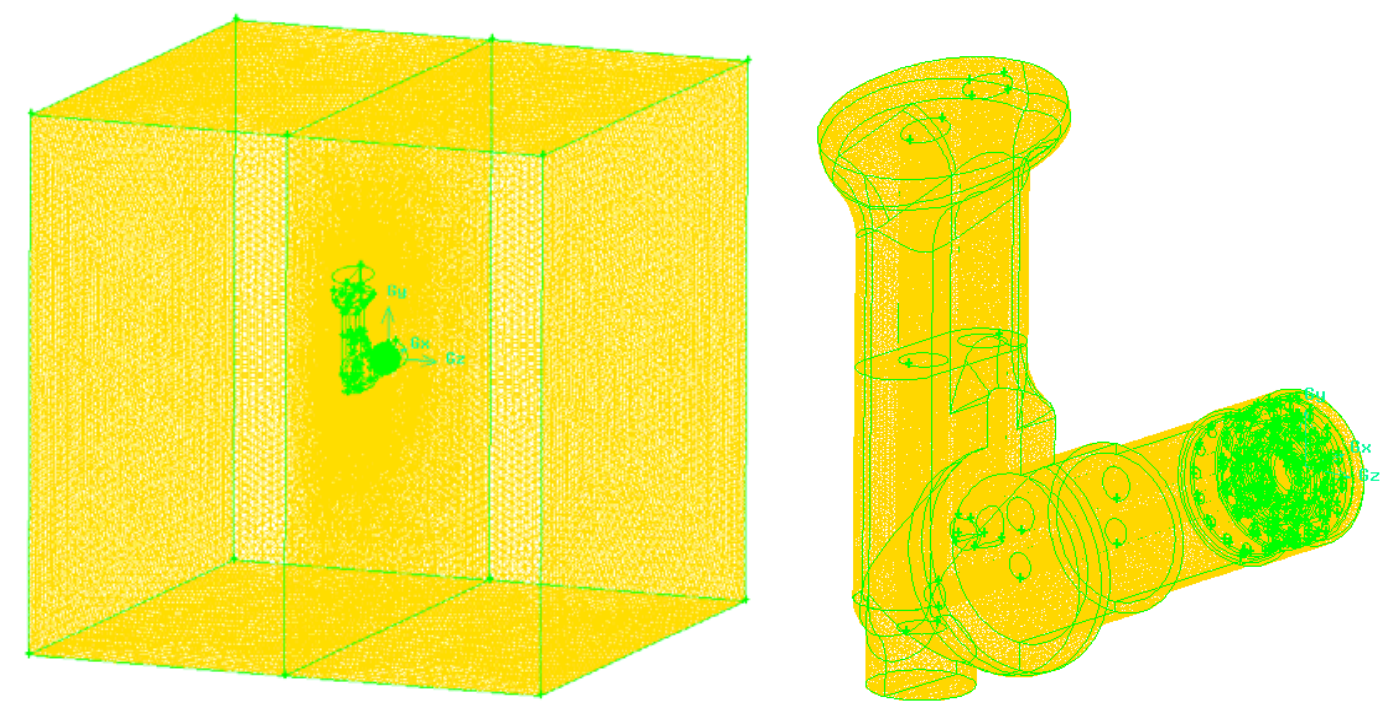

Figure 2. Schematic Diagram of calculation domain and global grid

\section{Simulation Result and Discussion}

The numerical simulation result of the typical working condition is acquired as what is shown in figure 3. According to the global temperature distribution and velocity distribution of the global flow field, the shell structure has high temperature difference with hot air. When the hot air flows around the shell structure, the shell is heated. The channel which has small flow is heated rapidly on account of closing to windward. The oil in channel which has high flow can cool the shell. The shell structure has high temperature in windward and low temperature in leeward. The oil in both channels has a temperature-rise from entrance to exit.

The main channel has high oil flow which can remove heat has lower temperature of oil than assistant channel. In flow and heat transfer process of fuel nozzle, the oil in main channel is always used as cold source which cools the shell srructure and the oil in assistant channel. The oil temperatuer of main channel is rising around the flow process and reaches acme at the outlet of main channel. The assistant channel which has low oil flow can remove heat at the first half of flow process and has warming effect on the main channel at the latter half. At first half of flow process, the oil in assistant channel is used as cold source to cool the shell structure and the temperature of oil in assistant channel rises. At latter half of flow process, the oil in assistant channel has higher temperature than that in main channel on account of less flow. In the alternative structure at the outlet of fuel nozzle, the oil in assistant channel is used as hot source to heat the main channel. In the flow process in assistant channel the oil temperature peak appears in the middle position of the channel. 




Global temperature distribution

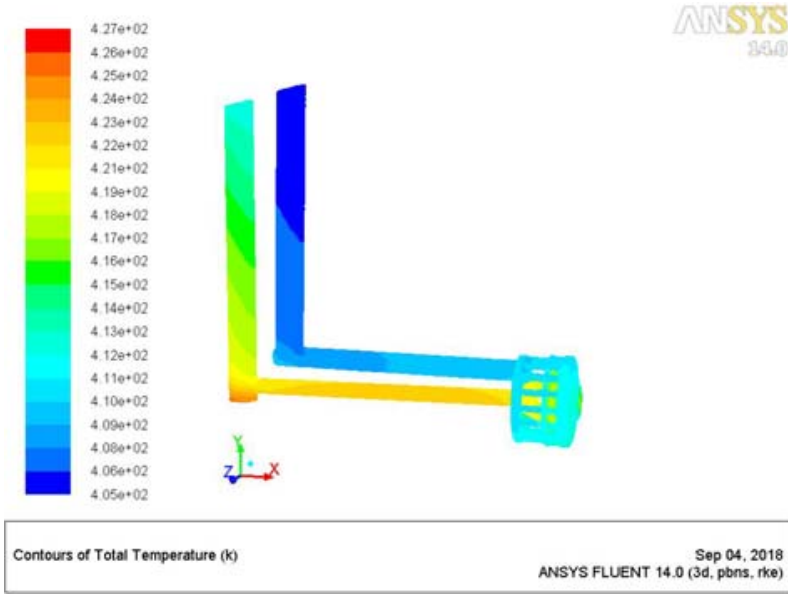

Fuel temperature distribution

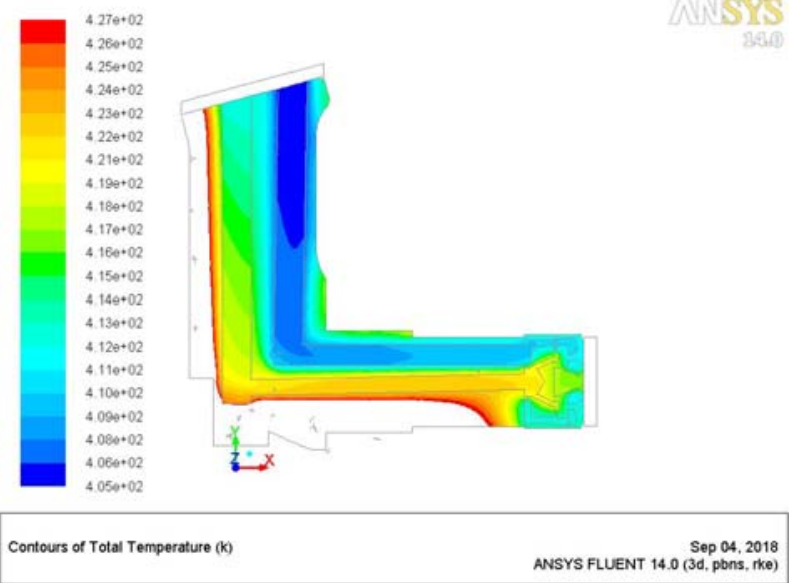

Center section temperature distribution
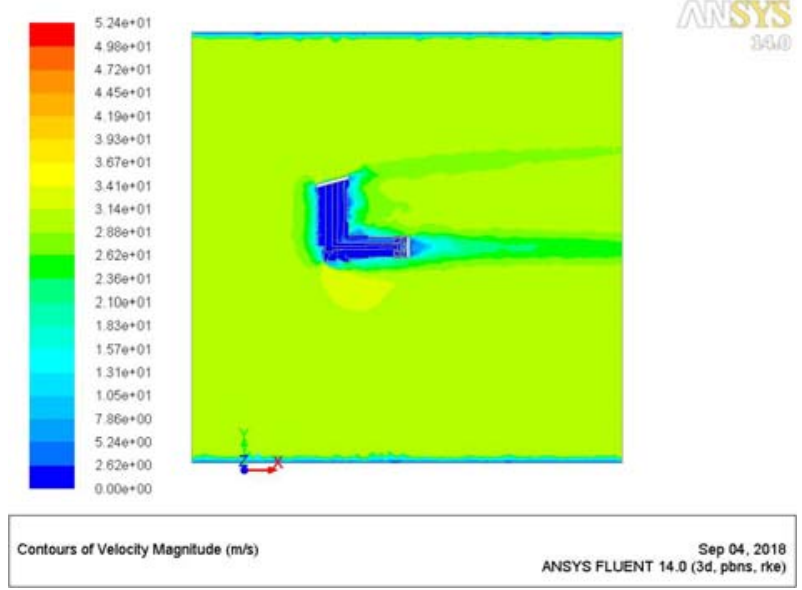

Global velocity distribution
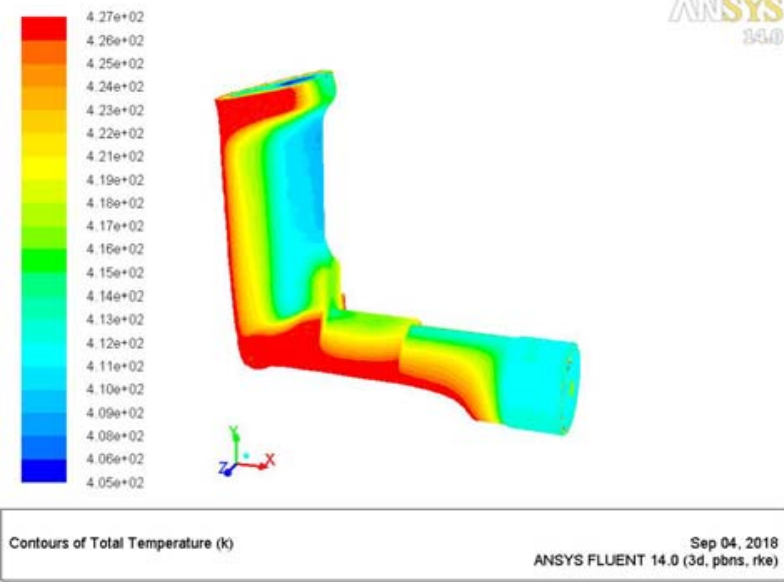

Nozzle temperature distribution



ANSYSFLUENT 140 Sep 04, 2010

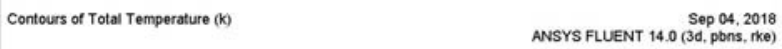

Outlet temperature distribution

Figure 3. Numerical simulation results

Based on the flow analysis above and the geomerry structuer of shell analysis, the high-occurrence position of coking can be confirmed. The main channel has the high-occurrence position of coking in outlet. The assistant channel has the high-occurrence position of coking in the middle of channel. The temperature of the position is selected as indicator to research coking characteristic of fuel nozzle with the change of flow parameter.

The figure 4 shows the variation trend of the highest temperature following the change of fuel delivery when aerodynamic parameter and oil temperature is constant. In general, the highest temperature of oil declines following the increase of fuel whether in main channel or assistant channel. The highest temperature of oil declines rapidly when the flux of assistant channel declines. The highest temperature of oil appears in the turning point of assistant channel. The change of flux 
has influence on the heat convection process directly. The change of flux in main channel has influence on the temperature distribution of shell structure first. Then, the shell heat the oil in assistant channel by heat conduction effect. The highest temperature of oil appears slow change following the increase of flux in main channel.

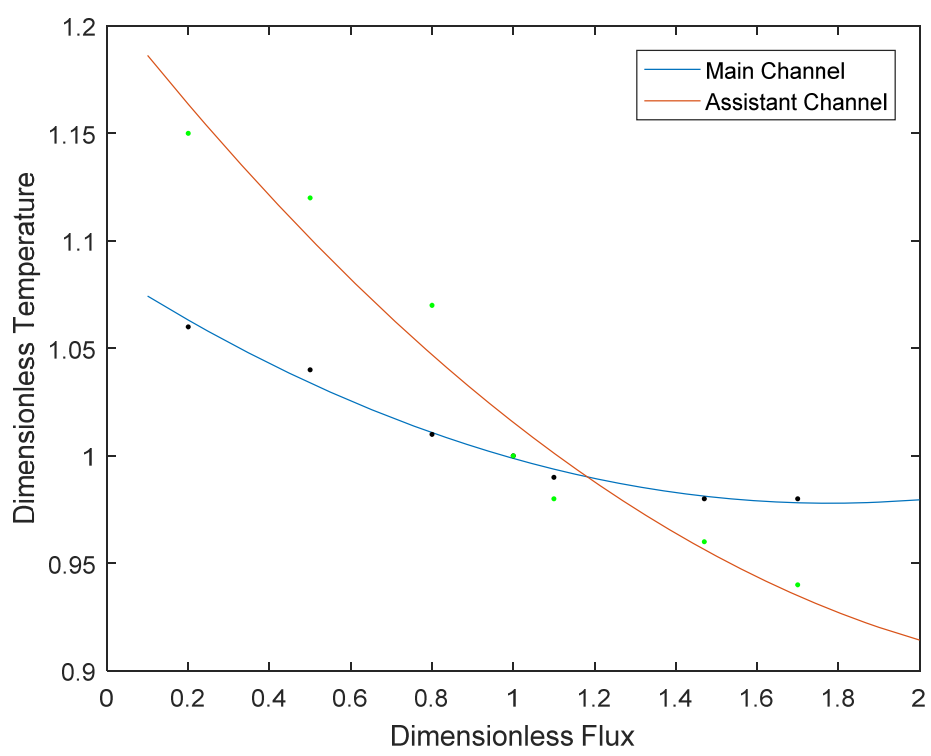

Figure 4. The variation trend of the highest temperature following the change of fuel delivery

The figure 5 shows the variation trend of the highest temperature following the change of aerodynamic parameter when fuel delivery and oil temperature is constant. In general, the highest temperature of oil increases following the increase of dimensionless temperature ratio and dimensionless velocity. The hot air is separate from the oil by shell structure. When the aerodynamic parameter changes the heat convection process between hot air and shell is impacted first and the heat conduction in shell structure is impacted immediately after.

The dimensionless temperature ratio increase means that the temperature difference between air and oil is increased. The high temperature difference can strong the the heat conduction process which results in more heat is transmited from air to oil. Based on the reason mentioned above the dimensionless temperature increases following the increase of dimensionless temperature ratio as what is shown in figure 5.

The dimensionless velocity reflect the change of air density and flux which play an important role in heat convection process. When the dimensionless velocity increasing the hot air flow around the shell structure increses accordingly. The heat convection process brings more heat to shell structure which change the temperatue of shell. Based on the reason mentioned above the dimensionless temperature increases following the increase of dimensionless velocity as what is shown in figure 5 .


Figure 5. The variation trend of the highest temperature following the change of aerodynamic parameter 


\section{Conclusion}

1. The high-occurrence position of coking in a fuel nozzle is determined by the geomerry structuer and flow process. The geomerry structuer has influence on the flow of oil and the heat conduction process. The flow process directly influents the heat convection process.

2. When aerodynamic parameter and oil temperature is constant, the highest temperature of oil declines following the increase of fuel whether in main channel or assistant channel.

3. When fuel delivery and oil temperature is constant, the highest temperature of oil increases following the increase of dimensionless temperature ratio and dimensionless velocity.

\section{References}

[1] Zhang Yingli, Chong Daotong, Liu Jiping, Yan Junjie. Numerical Investigation on Conjugated Heat Transfer of Conduction in Wall and Mixed Convection, Journal of Xi'an Jiaotong University, Vol.5, 2012.

[2] Jia Chunyan, Wang Hongming. Investigate on Fuel Coke in Nozzle of Aeroengine Combustor, Aeroengine, Vol.37, No.5, 2011.

[3] Chin J S, Lefebverv A H. Influence of flow conditions on deposits from heated hydrocarbon fuels, ASME 92-GT-114.

[4] Bilgen E. Conjugate heat transfer by conduction and natural convection on a heated vertical wall, Applied Thermal Engineering, Vol.29, 2009.

[5] Zhao Jianhua, Zhou Songlin, Lu Xianghui, Gao Dianrong. Numerical Simulation and Experimental Study of Heat-fluid-solid Coupling of Double Flapper-nozzle Servo Valve, Chinese Journal of Mechanical Engineering, Vol.5, 2015. 